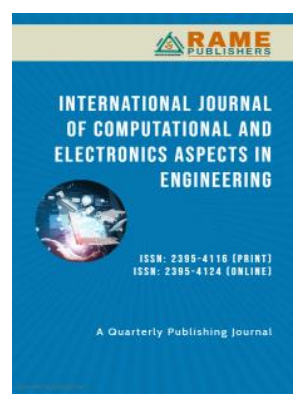

Ansar Sheikh

sheikh.ansar016@gmail.com

Shivani Makde shivanimakde0500@gmail.com

Bhuvaneshwari Gaddamwar bhuvigaddamwar@gmail.com

Khushbu Akare

Corresponding Author akarekhushbu9371@gmail.com

Poonam Thakur poonamthakur@gmail.com

Nikita Dongre nikitadongre1999@gmail.com

Sonali Deshmukh dsonali331@gmail.com

Shraddha Malwe shradhamalwe@gamil.com

${ }^{1}$ Assistant Professor, Department of Computer Engineering, Suryodaya College of Engineering and Technology, Nagpur, India

${ }^{2}$ Student, Department of Computer Engineering,

Suryodaya College of Engineering and Technology, Nagpur, India

\title{
A Review on Detection of Covid Symptoms
}

Abstract - Telemedicine may hold the key to limiting the emerging coronavirus disease's global outbreak (COVID-19). The COVID-19 virus directly affects the lungs, causing pneumonia-like symptoms and shortness of breath, both of which can be fatal. Despite the fact that self-quarantine and social isolation are crucial during a pandemic, the technique for identifying COVID-19 contraction via nose swabs, saliva test kits, and blood testing is routinely available at healthcare facilities. As a result, developing customized self-testing kits for the COVID-19 virus and related viruses is a top goal. Many e-health initiatives are now possible thanks to smartphones with embedded software, hardware, high-performance computation, and networking capabilities. COVID-19 contracted users' breathing sounds may reveal certain acoustic signal patterns that are worth researching, according to a thorough assessment of breathing sounds and their implications in recognizing respiratory issues. Obtaining respiratory data solely from breathing noises input into a smartphone's microphone appears to be an intriguing solution in this area. Advanced signal processing and analysis, in conjunction with modern deep/ machine learning and pattern recognition algorithms, can be used to analyze the obtained breathing sounds to separate the breathing phases, estimate lung volume, oxygenation, and further classify the breathing data input into healthy or unhealthy situations. For the ongoing global COVID-19 pandemic, the concepts mentioned have the potential to be employed as self-test breathing monitoring apps, with users being able to check their breathing sound pattern on a frequent basis.

Keywords - Sensor technology, e-health, telemedicine, and home health monitoring are all examples of technology.

\section{INTRODUCTION}

The COVID-19 virus has been labelled a pandemic by the World Health Organization (WHO), with more than 10

Review Article

Published on - 10 July 2021

(C) 2021 RAME Publishers

This is an open access article under the CC BY 4.0 International License https://creativecommons.org/licenses/by/4.0/

Cite this article - Ansar Sheikh, Shivani Makde, Bhuvaneshwari Gaddamwar, Khushbu Akare, Poonam Thakur, Nikita Dongre, Sonali Deshmukh, Shraddha Malwe, "A Review on Detection of Covid Symptoms", International Journal of Computational and Electronic Aspects in Engineering, RAME Publishers, vol. 2, issue 3, pp. 61-70, 2021.

https://doi.org/10.26706/ijceae.2.3.20210605 million infections and 503,862 fatalities globally as of June 30, 2020, according to WHO estimations. COVID-19 is caused by the extreme acute respiratory syndrome Coronavirus 2 (SARS-CoV-2) [1]. The World Health Organization labelled it a pandemic on March 11, 2020. The virus's probable genetic alterations may make COVID19 recovery even more difficult [2]. Billions of people are affected by the epidemic, which has resulted in significant changes in social relationships, health policy, trade, job, and educational environments. The global pandemic poses a grave threat to human civilization, necessitating immediate response [3]. The COVID-19 pandemic has 
motivated scientists to conduct cutting-edge research into viral mitigation, detection, and prevention in order to assist front-line medical personnel. The scientific community has been working on ideas to help contain the outbreak and prevent pandemics. Scientists leveraging developing technologies have created new techniques to tackle the pandemic, in addition to medical and virology expertise.

The new coronavirus illness (COVID-19) pandemic, which started in 2019, has posed a major threat to human life and health. This freedom helps interdisciplinary students, researchers, and professionals recognize COVID19 and reflect on perceptive intelligence, as well as monitor, regulate, avoid, anticipate, diagnose, and cure the condition in the future. Among the subjects covered in the book are fundamental knowledge, epidemic prediction models, surveillance and monitoring systems, integrated systems based on IoT and IoMT for COVID-19, social media analysis systems for COVID-19, radiological imaging (CT, X-ray-based diagnostic system) and computational intelligence, in silico drug creation, and drug reuse strategies against COVID-19. The contributors to this volume are experts in their disciplines who come from a wide range of famous colleges and institutions around the world. This volume is a comprehensive and useful resource for computer scientists and computer scientists, epidemiologists, radiologists, doctors, nurses, pharmacy practitioners, as well as graduate students and researchers in interdisciplinary and multidisciplinary sciences.

\section{HISTORY AND ORIGIN}

For the first time, the corona virus was labelled cold in 1960. Approximately 500 people were shown to have a flulike system in a research conducted in Canada in 2001. According to a polymerase chain reaction, 17-18 of them were infected with a corona virus strain. Corona was thought to be a harmless virus until 2002. Several reports showing the development of corona in the United States, Hong Kong, Singapore, Thailand, Vietnam, and Taiwan were published in 2003. In 2003, corona was linked to several episodes of extreme acute respiratory syndrome, with over 1000 people dying as a result. It was a horrible year for microbiologists. When the microbiologist begins to focus on these issues. After rigorous testing, they were able to determine and understand the disease's aetiology, and the virus was recognized as a corona virus. The corona virus infection, on the other hand, has been verified in 8,096 people thus far. The World Health Organization and the Centers for Disease Control and Prevention declared an "emergency" in 2004 as a result of this According to another store, a Hong Kong investigation found 50 patients with extreme acute respiratory syndrome, 30 of them were infected with the virus. In 2012, Saudi Arabian press reported on a number of infected patients and deaths [4-7]. For the first time, COVID-19 was found and isolated in pneumonia patent from Wuhan, China [8].

\section{Mode OF SPREADING}

It is possible to become infected by coming into close contact with someone who is showing signs of the virus, such as coughing and sneezing. Corona virus is disseminated primarily through the air by airborne droplets [9]. In the ciliated epithelium, the virus multiplied, causing cell death and infection at the infection site. A study released in 2019 revealed angiotensin converting enzyme 2 (ACE.2), a membrane exopeptidase in the receptor exploited by the corona virus when it penetrates human cells [10-12]. The WHO received notice of instances of pneumonia of unknown cause in China, East Asia's most populous area, on December 31, 2019. As of January 3, 2020, 44 instances of pneumonia had been documented. Chinese investigators said on January 7, 2020 that they had isolated a new virus named 2019-nCoV from the Wuhan city seafood market. On January 13, 2020, the Thai Ministry of Public Health confirmed that one patient had been imported from Wuhan, China. According to sources, the Japanese Ministry of Health, Labor, and Welfare was the first case imported from Wuhan to China on January 15, 2020. On January 20, 2020, Korea's national RSI focal point certified the first nCoV case of 2019. On January 23, 2020 , the first case of 2019-nCoV in the United States of America was verified. On January 24, 2020, Vietnam announced the first incidence of 2019-nCoV in a Chinese 
traveler's relative with no history of travel from China. As a result, the corona virus has been transmitted from person to person for the first time. On January 24, 2020, the Singapore government was confirmed as the first case of 2019-nCoV The Australian government, the Federal Democratic Republic of Nepal, and the French Republic were all revealed on January 25, 2020 for the first time in 2019-nCoV.

\section{RELATED WORK}

We looked into different specifics regarding the current application as part of the related work. They are given below: -

The following criteria were used to pick articles and apps discovered through search techniques. From 2019 to the present, both COVID-19-related citations and cell phone applications were examined. All prototypes, incomplete apps, and apps not available in an app shop were excluded due to the potential of spyware, malware, and viruses being disseminated via mobile apps downloaded from sources other than app stores. The citations from each search method were imported into an Excel spreadsheet, where the titles and abstracts were checked for inclusion or removal. Residents, health professionals, and policymakers consider mobile apps to be a significant tool in the face of the pandemic's critical difficulties, such as minimizing hospital overcrowding, receiving reliable information, and tracking symptoms and disease. People's mental health is being studied in innovative ways.

The Mawid application, which is a central appointment system, is provided by the Saudi Arabian Ministry of Health. Patients can use the app to plan, change, and reschedule appointments at any of Saudi Arabia's 2400 primary care centers, as well as manage referral appointments. Patients may use the app's GPS and mapping feature to see which primary care centers are nearest to them. They can go to any primary care institution and make appointments whenever they want. When an earlier appointment slot becomes available, the app frequently notifies the user, which might be useful for patients who require an early appointment [13]. A self-assessment function for COVID-19 is included in the framework, as well as a public consultation window. Patients must fill out a questionnaire for the self-assessment exam that includes information such as their travel history and symptoms, as well as other pertinent questions. At the time of its introduction in May 2019, the platform was embedded in 98 percent of hospitals and primary care centers, with over 6.5 million registered users. [14]. The application has provided consultation services to over half a million people and over 250,000 self-assessment tests have been registered since the COVID-19 epidemic in Saudi Arabia [15-16]. During the COVID-19 outbreak, the Mawid application, which was originally developed to promote digital health, proved to be quite effective in providing healthcare services.

With the aid of the Arizona Department of Health Services, the University of Arizona developed the COVID watch app (USA). The software is now in use at the institution, with ambitions to expand it across Arizona in phases. The app's goal is to alert users if they come into close proximity to someone who has been infected and has registered with it. The app communicates with you using Bluetooth technology and does not track your location [17]. In addition, no personally identifiable information about the user is gathered.

The PathCheck app (USA) is a contact tracing software developed by MIT and TripleBlind, who teamed up to form the Pathcheck Foundation, a new non-profit organization. The app's purpose is to link people and health-care facilities in order to share information and prevent the spread of the new coronavirus. The software makes advantage of Google's Apple Exposure Notification API, which assures that user data is kept private and secure. [nineteen] On their tablets, users can save their private location and symptom logs. The programme, which integrates various health systems, allows users to choose their chosen provider to share information and access services [19]. 
TraceTogether, a mobile touch tracing technology, was unveiled by the Singapore government. This software, like the Aarogya Setu app, employs Bluetooth technology to detect and inform users when they are in close proximity to an infected person. This programme uses anonymous identifiers that are sent back and forth between TraceTogether-enabled cellphones. [20] a Privacy and security were prioritized during the app's development. Bluetooth data on smartphones is automatically destroyed after 25 days. Additionally, users have the opportunity to delete data, maintaining excellent privacy standards [21, 22].

The Indian government developed Aarogya Setu, an official smartphone app, to track the COVID-19 epidemic and prevent it from spreading further. The software uses GPS and Bluetooth technology to keep track of things [23]. This app, like Tawakkalna, connects to neighbouring devices using Bluetooth technology and uses GPS to track the user's location. Other mobile users who have been hacked and utilize the same software as you have been. Self-assessment evaluations, test reports, electronic travel permits, COVID-19 information, precautionary approaches information, online queries, and more are all included in this package.[24]. The possibility of contamination has led to the creation of 697 coronavirus hot areas around the world. However, information collecting, intent restriction, data storage, administrative inconsistencies, a lack of laws, accountability, and auditability have all been raised as security problems in regard to data utilization[25]. The majority of individuals now utilise the app as a result of the government's solution to these challenges. This study focuses on the Aarogya Setu method, which was established by the Indian government. This is a smartphone application developed by the Ministry of Health as part of an EGovernance programme to track and educate Indians about COVID-19. The purpose of this study is to learn about some of the tool's useful features and to showcase numerous applicable data science principles found in the app, as well as their importance in pandemic management. The software uses Bluetooth and GPS technology to warn users when they are near someone infected with COVID-
19. The programme uses several data science principles such as classification, association rule manipulation, and grouping to investigate the distribution of COVID-19 in India. The research will benefit mobile technology experts, data science professionals, clinicians, frontline healthcare workers, public officials, and civil servants.

The authors suggest a scoping analysis for mobile apps for Covid-19. This study evaluates the purpose of COVID19-related applications as well as the methods used by these apps. 9 makes conclusions concerning the needs that such application-based solutions fail to address [26]. Government, healthcare organisations, and application developers must collaborate and innovate to address the weaknesses identified and encourage the successful use of mobile apps in the administration of COVID-19.

The Australian Department of Health developed the COVID Safe application, which is a contact-tracing smartphone app. It has been certified by the Australian government as the sole mode of communication. The purpose of the app is to track people's movements and see if they've come into contact with infected folks. The app provides users a notification if they do [27]. Health officials will also contact you and offer you with the appropriate help and information. To safeguard their identity, users can register under a pseudonym and provide their cell phone number. Allowing the app to operate in the background is recommended. It uses Bluetooth technology to communicate with other devices that have the COVID Safe application loaded (using a digital handshake). Date, time, and phone numbers are safely 4 encrypted and stored on the phone for 21 days; after that period, users can erase the data. Furthermore, the data stored in the National COVID Safe Data database will be lost after the conclusion of the epidemic [28].

Tawakkalna has been authorised as an official mobile app by the Saudi Arabian Ministry of Health. The goal of this software is to make it easier for individuals to travel around during a lockdown or curfew in an emergency. The programme also includes COVID-19 data, such as the number of illnesses in various places. In the event of an 
emergency during curfew, Saudi citizens can utilise the app to ask for travel permissions. If users are in close proximity to contagious or toxic places, they may receive notifications [29]. This is a mock-up of a Chinese app that represents the user's current position using a color-coded QR code. Green indicates that the individual is in good health and has been granted permission to fly. Because they are suspected of possessing COVID-19, the person is not allowed to travel. The colour red indicates that the person is ill, unable to fly, and needs to be quarantined [30].

Immuni, a contact tracing system, was launched by the Italian government in June 2020. Like previous contact tracing apps, this app notifies users whenever they come into 6 close touches with someone who is using the same programme and has been detected as infected. The app's distinguishing characteristic is that it makes use of Bluetooth technology that consumes very little power. Furthermore, no personally identifying information such as a user's name, date of birth, location, phone number, or email address is collected by the app. When two cellphones with the Immuni app installed come into close proximity, the produced codes are automatically transferred [31]. If any of the users has been diagnosed with the virus, the software can recognise past interactions. With the patients' agreement, the health authorities enter the code into a centralised system, which is subsequently used to inform any users who have been into close contact with the patient (using the code) [32]. Furthermore, because the software is multilingual, it may be used in Italy by people from all over the world. The software does not track users' movements and solely provides tracking codes for contacts. Before December 31, 2020, all data gathered and exchanged with the central server will be destroyed.

The Tabaud app was developed to help Saudi Arabia meet health and safety goals by containing the COVID-19 outbreak and reducing the transmission of the novel coronavirus, hence easing limitations on social and commercial activities[33] .This software uses the Google Apple API to monitor contacts, ensuring that the user's privacy and security are safeguarded. As a result, rather than employing position maps, the software employs Bluetooth technology to detect nearby devices that are running the same programme. If a user reports themselves as infected on the app, the information is sent to the Health Department for verification. If the ministry validates the information, all Tabaud app users who were within 14 days of the sick individual would receive a notification to take the necessary safeguards. The software also looks at the sick person's proximity to other cellphones to determine their vulnerability and risk of infection. It also allows users to assess their risk of infection. By sending the person's disguised identifying data while engaging with other mobile applications and adhering to rigorous privacy standards, people's identities are obscured and anonymity is retained. More than 15,000 positive cases have been reported by patients since the app's introduction in June 2020 [34]. The software was successful in locating contacts and limiting the spread of the new chorus.

The developers have proposed a comprehensive survey for the open source covid-19 dataset. This article provides a thorough examination of open source COVID-19 data sets. Based on the type of data and how it was used, the survey was separated into sections. The most common categories of data were medical images, text data, and voice data. COVID-19 diagnosis, infection prediction, mobility and demographic correlations, NPI analysis, and sentiment analysis were all done with the open-source dataset. They discovered that there is space for open-source data curation and recovery in a variety of methods, including increasing sets, despite the fact that scientific study on COVID-19 is ongoing and quickly expanding. Data from CT tomography that can be utilized to collect cough placement data using deep learning techniques. They compared and contrasted the workers in terms of their start-up, deployment, and machine learning (ML)/statistical approaches. They also talked about potential research avenues and roadblocks for COVID-19 data sets that are open source. They say that the primary difficulty of data-driven $\mathrm{AI}$ is the opacity of data and analytic methodologies. More work is needed on conserving the COVID-19 dataset for cough diagnosis, 
expanding the CT and X-ray data sets for more precision in learning in-depth methodologies, building frameworks for patient data privacy security, and user interface design. The importance of mobility and contacts cannot be overstated. Monitoring, non-contact diagnostics based on biomedical imaging to protect frontline healthcare workers are only a few examples. Just a few instances include sentiment analysis and false re-identification of social media for policymaking, as well as semantic analysis for automated discovery based on scientific article content. They claim that the effort stated in this open source and data-driven survey is the way to a COVID-19 scientific study that is scalable, clear, and verifiable [35].

The COVID-19 pandemic, according to the author of the COVID-19 contact tracing programme, continues to have an impact on everyone's way of life. Contact tracking methods will almost probably be crucial in assisting health officials in identifying persons who have been exposed to the virus as quickly as possible. Growing interest in and acceptance of tracking application technologies may assist health authorities in expanding their tracking capabilities; however, as this article points out, this is not a simple cure. Users, data protection agencies, and researchers are all concerned about these applications. The processing of user data, the likelihood of non-trivial false positives and negatives, as well as the security and privacy of the applications, are all considerations to consider. This article offered an overview of the three most prevalent monitoring framework architectures: centralised, decentralised, and hybrid, as well as an overview of popular applications in these categories, based on these concerns. The study also addressed privacy and security concerns, outlining the different types of attacks that may be used against each of the three architectures. Other users' questions about battery use, compatibility, withdrawal of consent, and transparency are addressed in this report [36].

The authors based their findings on the existing condition of coronavirus illness. The COVID-19 epidemic has posed a danger to practically every business as the disease 10 spreads at an unprecedented rate around the globe COVID-19, in particular, is an RNA virus that can be harmful to people's health. Many of individuals have been infected with the sickness, and thousands more have died as a result of it. In principle, the disease's rapid spread demands methods to prevent future spread following a comprehensive investigation and quarantine. In general, no effective drugs or vaccinations have been discovered to improve the health of illness patients. Isolation, sufficient ventilation, hand cleanliness, and the use of personal protective equipment, such as surgical masks, goggles, gloves, and gowns, are all recommended for infection prevention [37].

NHS Test and Trace has released the NHS COVID-19 app in the United Kingdom. The software uses low-power Bluetooth technologies and GPS to track the user's travels. This programme does a number of tasks. First, it will issue a warning whenever a user comes into close contact with other registered app users who have tested positive for COVID-19. Second, consumers can register for a free trial of COVID-19 by registering their symptoms. Finally, users who sign up for a place or location will be notified if the place or location has a big number of positive cases. [38] It also allows users to track their self-isolation timer and access useful advice. The app does not save any personally identifiable information that may be used to identify a consumer. However, information such as location monitoring, zip code, and registration is collected [39].

The COVID Symptom Study app was developed in collaboration with researchers from King's College London's Guys, St Thomas Hospitals, and Zoe Global Limited, a healthcare technology firm. The purpose of this software is to track the spread of the virus, identify highrisk areas in the UK, identify vulnerable social groupings, and recognize symptoms associated with underlying medical issues. [40] Its goal, on the other hand, is to gather data from people in order to aid the COVID-19 study in the United Kingdom and improve preventive measures. measures. People will volunteer to participate in the study and will exchange two sorts of data on a regular basis. The first section contains general information such as age, fitness, and underlying conditions, while the second section contains information on symptoms [41]. More than 4 
million people in the UK have volunteered to give data through the app, resulting in a database that may be used to determine COVID-19 expertise [42].

The authors proposed a COVID-19 infection in their paper: disease detection and mobile technologies. The coronavirus is believed to have started in a Hunan seafood market in Wuhan, China, and quickly spread to 215 nations. Despite the fact that various clinical trials for vaccine availability and treatment therapies have commenced, no authorized evidence-based vaccinations or treatments for the treatment of human coronaviruses, notably Covid 19, are now available. COVID-19 treatment techniques are now being developed by scientists and researchers. Several organizations are striving to create viable SARS CoV-2 vaccines, but following active human and animal studies, commercialization takes months. In the meanwhile, it's critical to prevent the infection from spreading. Mobile technology, notably app-based technology, is helping to track virus propagation and provide information on best-practices in mitigations such self-quarantine in a number of nations. In their study, the authors proposed a COVID-19 infection: disease detection and mobile technology. These apps are simple to use, and when the literature begins to report on the technology's success, their widespread acceptance is anticipated to expand (Joshi, 2020). As the saying goes, "a crisis presents an opportunity." The first major crisis of 2020 gives an opportunity to build best practices in the use of mobile technology for healthcare, as the adage goes. For chronic illnesses like diabetes and hypertension, the potential benefits of digital app-based healthcare therapies appear to be especially compelling [43-44].

TABLE I

LITERATURE SURVEY

\begin{tabular}{|c|c|c|c|c|}
\hline Sr. No & Title & Agency & Methodology & Conclusion \\
\hline 1 & Trace Together & $\begin{array}{l}\text { Government Technology } \\
\text { Agency (Govtech) In } \\
\text { Collaboration with The } \\
\text { Ministry of Health (Moh) }\end{array}$ & $\begin{array}{l}\text { Trace Together Is a Popular Smartphone } \\
\text { Contact Tracking App That Uses Bluetooth to } \\
\text { Track the Infected People and Alert People } \\
\text { Who Have Been Close to Then }\end{array}$ & $\begin{array}{l}\text { The Main Idea of This Paper Was to Build a } \\
\text { Software to Detect the Covid Patient }\end{array}$ \\
\hline 2 & $\begin{array}{l}\text { NHS smartphone } \\
\text { App }\end{array}$ & $\begin{array}{l}\text { British National Health } \\
\text { Service }\end{array}$ & $\begin{array}{l}\text { The app will keep track of individuals' } \\
\text { travels and notify individuals who come into } \\
\text { contact with sick persons. According to the } \\
\text { NHS, the app could aid in the relaxation of } \\
\text { lockdown by evaluating virus distribution } \\
\text { patterns and hotspots. }\end{array}$ & $\begin{array}{l}\text { The software would categorize user } \\
\text { information based on demographics, living } \\
\text { arrangements, and transportation habits. A } \\
\text { maximum number of persons might be determined } \\
\text { based on the data analysis and allowed to travel } \\
\text { freely. }\end{array}$ \\
\hline 3 & Covid Watch & Stanford University & $\begin{array}{l}\text { It detects users when they are close by } \\
\text { using Bluetooth signals and notifies them } \\
\text { anonymously if they have been in contact with } \\
\text { someone who has tested positive. }\end{array}$ & $\begin{array}{l}\text { Any third party, including the government, will } \\
\text { be unable to monitor who has been exposed by } \\
\text { whom, which is a unique feature of the app. }\end{array}$ \\
\hline 4 & $\begin{array}{l}\text { COVID symptom } \\
\text { Tracker }\end{array}$ & St. Thomas Hospitals & $\begin{array}{l}\text { To create this software, scientists looked at } \\
\text { high-risk areas in the United Kingdom, the rate } \\
\text { of virus propagation, and the most vulnerable } \\
\text { demographics based on health problems. }\end{array}$ & $\begin{array}{l}\text { This software records virus symptoms among } \\
\text { users and records virus symptoms for continuing } \\
\text { study. The app adheres to the General Data } \\
\text { Protection Regulation, and the information is only } \\
\text { used for health research and not for commercial } \\
\text { gain. }\end{array}$ \\
\hline
\end{tabular}


Furthermore, one author reported on the COVID-19 selftest on a smartphone using breath sounds. Self-testing for COVID-19 using respiration would be especially useful for those at higher risk of significant disease (for example, the elderly or patients with substantial underlying illnesses), as well as high-risk clinical cohorts of patients who can conduct quick and routine daily self-tests. Many people who come into contact with the public (such as traffic officers and police / force / emergency medical services (EMS) personnel) are at risk of contracting an infection. People who work in cultural settings (for example, multi-family families with numerous household members working in high-risk exposure scenarios) are also at risk. COVID-19 is a human-infectious virus [45]. Students, as well as those working in meatpacking factories or in front-line grocery store positions, are especially at risk. This emphasizes the need of using the breath sounds database to create breath application test models [46-47].

\section{CONCLUSIONS}

Using a mobile microphone to obtain respiratory data merely from amplified breath sounds appears to be an intriguing alternative. The goal is to develop an interactive breathing simulation framework for self-diagnosing respiratory problems using a smartphone. Furthermore, the software enhances lung function by displaying highdefinition Virtual lung animations that inflate and deflate in real time when the user inhales and exhales. As a result, to represent lung volume and oxygenation, we advocate using respiratory sound models. Mel's frequency analysis can be used to understand breath sounds that have been captured. Using new pattern recognition and deep / machine learning algorithms, separate breathing phases and quantify lung capacity and oxygenation (e.g. recurrent neural networks). Once the database is built, our artificial intelligence systems will be able to connect these data with breath sounds. Using regression models, annotated samples were utilised to estimate lung capacity and oxygenation of breath sounds. Respiratory data could be classified as safe or unhealthy using AI classification methods (i.e., corresponding to a respiratory disease or a complication like pneumonia or COVID-related disease).

\section{REFERENCES}

[1] Organization. Summary of probable SARS cases with onset of illness from 1 November 2002 to 31 July 2003.

[2] World Health Organization. Coronavirus disease 2019 (covid-19): situation report, 162. 2020.

[3] Matt J Keeling, T Deirdre Hollingsworth, and Jonathan M Read, "The efficacy of contact tracing for the containment of the 2019 novel coronavirus (covid-19)", medRxiv, 2020.

[4] Stefano Boccaletti, William Ditto, Gabriel Mindlin, and Abdon Atangana, "Modeling and forecasting of epidemic spreading: The case of covid-19 and beyond", Chaos, Solitons, and Fractals, 2020.

[5] Centers for Disease Control and Prevention (CDC), "Update: Outbreak of severe acute respiratory syndrome--worldwide, 2003”, MMWR Morb Mortal Wkly Rep. 2003; 52(12):241-6.

[6] World Health Organization. Coronavirus never before seen in humans is the cause of SARS- update 31. Geneva: The Organization; 2003.

[7] World Health Peiris JS, Lai ST, Poon LL, Guan Y, Yam LY, Lim W, et al. Coronavirus as a possible cause of severe acute respiratory syndrome. Lancet. 2003; 361:1319-25.

[8] World Health Organization. WHO Statement Regarding Cluster of Pneumonia Cases in Wuhan, China Geneva 2020, updated 9 January 2020 and 14 January 2020. Available athttps://www.who.int/china/news/detail/09-01-2020-whostatement-regarding-cluster-of-pneumonia-cases-in-wuhanchina

[9] Zhu N, Zhang D, Wang W, Li X, Yang B, Song J, et al., “A Novel Coronavirus from Patients with Pneumonia in China, 2019”, New England Journal of Medicine, 24 January 2020.

[10] Woo PC, Lau SK, Huang Y, Yuen KY., "Coronavirus diversity, phylogeny and interspecies jumping”, Exp Biol Med (Maywood), 2009 Oct; 234(10):1117-27. doi: 10.3181/0903-MR-94.

[11] De Souza Luna LK, Heiser V, Regamey N, Panning M, Drexler JF, Mulangu S, et al., "Generic detection of coronaviruses and differentiation at the prototype strain level by reverse transcription-PCR and nonfluorescent lowdensity microarray”, J Clin Microbiol, 2007 Mar; Volume 45(3):1049-52. doi: 10.1128/JCM.02426-06. Epub 2007 Jan 17 
[12] Letko M, Munster V., "Functional assessment of cell entry and receptor usage for lineage $\mathrm{B} \beta$-coronaviruses, including 2019- nCoV. bioRxiv", Nature Microbiology doi: 10.1038/s41564-020-0688-y.

[13] Sharma T, Bashir M.. "Use of apps in the COVID-19 response and the loss of privacy protection", Nat Med. 2020; Volume 26(8):1165-1167.

[14] Ministry of Health. E-Services - (Mawid) service; 2020. Available from: https://www.moh.gov.sa/en/eServices/Pages/cassystem.aspx. 20, 2020.

[15] Frontier Enterprise. New app from Saudi Ministry of Health makes access to health services easier; 2020. Available from: https://www.frontier-enterprise.com/new-app-from-saudiministry-of-health-makes-access-to-health-services-easier/. Accessed August 20, 2020.

[16] Arab News. Saudi Arabia's Mawid smartphone app offers coronavirus self-assessment; 2020. Available from: https://www.arabnews.com/node/1652171/saudi-arabia.

Accessed August 20, 2020.

[17] Covid Watch. COVID Watch exposure notification app digital contact tracing; 2020. Available from: https://www.covidwatch.org/. Accessed August 20, 2020.

[18] Pathcheck Foundation. PathCheck Foundation | COVID-19 Technology \& Research; 2020. Available from: https://pathcheck.org/. Accessed August 20, 2020.

[19] Google Play. PathCheck SafePlaces; 2020. Available from: https://play.google.com/store/apps/details?id=org.pathcheck. covidsafepaths\&hl=en_US. 20, 2020.

[20] Stevens H, Haines M, “Tracetogether: pandemic response, democracy, and technology", East Asian Science, Technology Society. 2020.doi: 10.1215/18752160-8698301. $14523-532$

[21] Dhār T, "Aarogya Setu - Carrying your privacy in your hands?", May 29, 2020. Available from: https://ssrn.com/abstract=3614506. Accessed August 20, 2020.

[22] Mitchell WB, Mehta S., "Trace Together. Harvard business school case 820-111", 2020. Available from: https://www.hbs.edu/faculty/Pages/item.aspx?num=58357. Accessed December 30, 2020.

[23] Jhunjhunwala, "A. Role of telecom network to manage COVID-19 in India: Aarogya Setu", Transactions Indian National Academy Engineering. 2020; 5(2):157-161. doi:10.1007/s41403-020-00109-7
[24] Alam T., "Coronavirus disease (COVID-19): reviews, applications, and current status", SSRN Electronic J. 2020. doi:10.2139/ssrn.3660497

[25] Times of India.Aarogya Setu app download: Aarogya Setu app enters 100 million users club; 2020. Available from: https://timesofindia.indiatimes.com/gadgets-news/aarogyasetu-app-enters-100-million-usersclub/articleshow/75709726.cms. Accessed August 20, 2020.

[26] Australian Government. COVIDSafe app. Available from: https://www.covidsafe.gov.au/. Accessed August 20, 2020.

[27] Department of Health. COVIDSafe app. Available from: https://www.health.gov.au/resources/apps-andtools/covidsafe-app. Accessed August 20, 2020.

[28] Saudi Press Agency. SDAIA launches Tawakkalna app to facilitate the issuance of movement permits electronically during the curfew period. Official Saudi Press Agency; 2020. Available from. https://www.spa.gov.sa/viewfullstory.php?lang=en\&newsid= 2082059.

[29] Alam T., "Internet of things and blockchain-based framework for Coronavirus (COVID-19) disease", SSRN Electronic J. 2020. doi:10.2139/ssrn.3660503

[30] Google Play. Immuni; 2020. Available from: https://play.google.com/store/apps/details?id=it.ministerodell asalute.immuni\&hl=en. 20, 2020.

[31] Presidenza del Consiglio dei Ministri. Immuni; 2020. Available from: https://www.immuni.italia.it/. 20, 2020.

[32] Saudi Data and AI Authority. TABAUD; 2020. Available from: https://tabaud.sdaia.gov.sa/IndexEn. 20, 2020.

[33] Salama S. COVID-19: 15,000 Saudis report having coronavirus via app. Gulf News. 2020. Available from https://gulfnews.com/world/gulf/saudi/covid-19-15000saudis-report-having-coronavirus-via-app-1.73253086. Accessed, 2020.

[34] World Health Organization. Available at https://www.who.int/ (last accessed

[35] Lung Cancer Fact Sheet, American Lung Association. Available at www.lung.org (last accessed May 22, 2020).

[36] Brown MS, Goldin JG, Suh RD, McNitt-Gray MF, Sayre JW, Aberle DR, "Lung micronodules: Automated method for detection at thin-section CT-initial experience", Radiology 2003; Volume 226, 256-262.

[37] NHS UK. The NHS test and trace app support website; 2020. Available from: https://covid19.nhs.uk/. Accessed August 20, 2020. 
[38] NHS UK. Category · COVID-19 app support; 2020. Available from:

https://faq.covid19.nhs.uk/category/?id=CAT-

01022\&parentid=. Accessed August 20, 2020.

[39] The Local. Italy launches Immuni contact-tracing app: here's what you need to know. Available from: https://www.thelocal.it/20200605/italy-to-begin-testingimmuni-contact-tracing-app-in-four-regions. Accessed August 20, 2020.

[40] Sudre C, Lee K, Lochlainn M, et al., "Symptom clusters in COVID19: a potential clinical prediction tool from the COVID symptom study app", Sci Adv, 2021 Mar 19;7(12):eabd4177. doi: 10.1126/sciadv.abd4177.

[41] Zoe. COVID symptom study - help slow the spread of COVID-19; 2020. Available from: https://covid.joinzoe.com/. Accessed August 20, 2020.

[42] Zargar A. 2020. Privacy, security concerns as India forces virus-tracing app on millions. Available at https://www.cbsnews.com/news/coronavirus-india-contacttracing-app-privacydata-security-concerns-aarogya-setuforced-on-millions/.
[43] Zhong N, Zheng B, Li Y, Poon L, Xie Z, Chan K., "Epidemiology and cause of severe acute respiratory syndrome (SARS) in Guangdong, People's Republic of China, in February", Sci Adv, 2021 Mar 19;7(12):eabd4177, doi: 10.1126/sciadv.abd4177.

[44] Reichert S, Gass R, Brandt C, Andre`s E, "Analysis of respiratory sounds: State of the art", Clin Med Circ Respirat Pulm Med 2008; Volume 2, 45-58.

[45] Abushakra A, Faezipour M., "Acoustic signal classification of breathing movements to virtually aid breath regulation", IEEE J Biomed Health Inform, 2013; Volume 17:493-500.

[46] Abushakra A, Faezipour M., "Estimating lung capacity from acoustic signal of respiration:, IEEE EMBS Conference on Micro and Nanotechnology in Medicine. IEEE, Ka'anapali, HI, USA (IEEE MNM'12). 2012;19.

[47] Voorhees E, Alam T, Bedrick S, Demner-Fushman D, Hersh WR, Lo K, Roberts K, Soboroff I, Wang LL, "TRECCOVID: constructing a pandemic information retrieval test collection", 2020. arXiv:2005.04474. 